\title{
Succinylcholine and Morbid Obesity: The Debate Continues...
}

\author{
Jay B. Brodsky • Harry J. M. Lemmens
}

Received: 16 August 2009 /Accepted: 22 September 2009/Published online: 8 October 2009

(C) Springer Science + Business Media, LLC 2009

Despite its potential adverse side effects, succinylcholine has been used in clinical anesthetic practice for more than 50 years. Its benefits include rapid onset $(<1 \mathrm{~min})$ with excellent tracheal intubating conditions and rapid recovery from paralysis [1]. Although potential replacements for succinylcholine have been sought, no other neuromuscular blocking agent has yet been equal to succinylcholine in its onset time and time to full recovery from block [2].

Currently, the agent deemed most likely to replace succinylcholine is rocuronium. Although its onset to paralysis is almost equivalent to succinylcholine, rocuronium has a much longer (30-50 min) duration of action [3]. For a normal weight surgical patient with a normal airway and a long safe apnea time (time from paralysis until oxyhemoglobin desaturation), rocuronium can be substituted for succinylcholine. This is not the case for the morbidly obese patient in whom rapid access to a potentially difficult airway under the best possible laryngoscopy conditions is always necessary [4].

In several studies, it has been demonstrated that although rocuronium and succinylcholine are equivalent in achieving "good" intubating conditions, for consistently excellent conditions, succinylcholine is always a better choice [5]. Because of its short duration of action, in our opinion, succinylcholine is indicated for every morbidly obese

\footnotetext{
J. B. Brodsky $(\bowtie) \cdot$ H. J. M. Lemmens

Department of Anesthesia, H-3580,

Stanford University Medical Center,

300 Pasteur Drive,

Stanford, CA 94305, USA

e-mail: Jbrodsky@stanford.edu
}

patient since laryngoscopy may not always be successful, mask ventilation can be challenging, and safe apnea time is very short, increasing the need for rapid emergence from the block.

The reversal agent sugammadex can decrease the duration of action of rocuronium, and its release into clinical practice has led some to state that a replacement for succinylcholine has finally been found [1]. However, sugammadex is not available in the USA. In Europe where sugammadex is currently in use, a single reversal dose costs several hundred dollars (M. Margarson, Chichester, UK, personal communication) compared to succinylcholine which is very inexpensive.

Although the more serious side effects of succinylcholine (significant hyperkalemia, anaphylaxis, malignant hyperthermia) can be life-threatening, their occurrence is extremely rare. The more common side effects of succinylcholine are usually minor, and in our opinion, the benefits of succinylcholine in the morbidly obese surgical patient far outweigh any reason not to use it.

\section{References}

1. Lee C. Goodbye suxamethonium! Anaesthesia. 2009;64:73-81.

2. White PF. Rapacurium: why did it fail as a replacement for succinylcholine? Br J Anaesth. 2002;88:163-5.

3. McCourt KC, Salmela L, Mirakhur RK, et al. Comparison of rocuronium and suxamethonium for use during rapid sequence induction of anaesthesia. Anaesthesia. 1998;53:867-71.

4. Lemmens HJM, Brodsky JB. The dose of succinylcholine in morbid obesity. Anesthesiology. 2006;102:438-42.

5. Perry JJ, Lee JS, Sillberg VAH, et al. Rocuronium versus succinylcholine for rapid sequence induction intubation. Cochrane Database Syst Rev. 2008;2:CD002788. 